\title{
Jaqueca y equivalente convulsivo
}

\author{
DRES.: RICARDO GUZMAN C. *, RICARDO GUZMAN Y. *.
}

La Jaqueca o Migraña es una entidad descrita clínicamente desde hace ya largo tiempo, que ha sido ampliamente estudiada en sus bases anatómicas y fisiopatológicas (2-6-8-10-15). Este cuadro que se inicia generalmente en la infancia (1-11-17), ha sido definido como "un trastorno familiar caracterizado por crisis recurrentes de dolor de cabeza de intensidad, frecuencia y duración variables. Casi siempre estas crisis son unilaterales y asociadas con anorexia, náuseas y/o vómitos. En algunos casos están precedidas o acompañadas por trastornos neurológicos". (Definición del grupo de expertos de la Federación Mundial de Neurología, 1969).

La primera descripción de una jaqueca corresponde a Hipócrates. Areteo de Capadocia fue el primero en aislarla como una enfermedad autónoma separándola de otros tipos de cefalea. Galeno emitió la primera hipótesis patogénica y fue también él quien la denominó hemicránea.

Las teorías vasculares sobre la jaqueca aparecen con Wepfner en el siglo XVII. Precursores de las actuales hipótesis son los trabajos de la Escuela Americana de Graham, Wolff y Friedman. En la Escuela Alemana sobresalen los de Heyck. Ostfeld es uno de los primeros en plantear la teoría "vascularhumoral", perfeccionada por los aportes de Espadaler Medina y Sicuteri, actualmente en boga.

Equivalente Convulsivo es un concepto introducido por Waggner y Bagchi en 1947, quienes describieron 15 casos de cefaleas paroxísticas con electroencefalogramas anormales, denominándolas cefaleas epilépticas.

En 1947 Livingstone analizó 14 casos con

* Depto. Cefaleas. Servicio de Medicina. Hosp. San Juan de Dios. sintomatología similar. Halpern y Bental en 1958, y Espadaler Medina en 1961, siguieron redondeando el concepto.

Para algunos autores, el Equivalente Convulsivo sería una Epilepsia ( 3 - 4 - 13). La forma más frecuente de expresión en este tipo de epilepsia, serían las crisis de cefalea periódica, acompañadas de signos colaterales tales como dolores abdominales, náuseas y/o vómitos. Algunos pacientes tienen antecedentes de crisis convulsivas (3-5 - 13).

Como puede apreciarse, las dos entidades anteriormente mencionadas presentan una sintomatología básica similar que lleva a plantear algunas interrogantes:

- Jaqueca y equivalente convulsivo, ¿corresponden a una misma enfermedad?

- ¿Sería la jaqueca un tipo de epilepsia?

- ¿Qué relaciones o diferencias pueden establecerse entre ambos cuadros?

Estas preguntas no han encontrado una respuesta clara en los diferentes estudios sobre el tema y son numerosas las opiniones a favor o en contra de esta asociación (3-57-10-12-13).

Nuestro trabajo no pretende hacerlo en forma definitiva, lo que estaría lejos de nuestras posibilidades; pero sí, busca precisar en la medida que nos sea posible, los límites de ambos cuadros, resaltando la importancia de realizar un diagnóstico diferencial correcto.

MaterJal y metoino. Se seleccionaron 31 niños, 19 con diagnóstico de jaqueca y 12 de equivalente convulsivo, de un total de 45 escolares estudiados prospectivamente en el Depto. de Cefaleas, Hospital San Juan de 
Dios Todos ellos consultaron por presentar cefalea pertinaz y recurrente que no cedia a los analgésicos habituales y cuyas crisis se habian iniciado hacía por lo menos un año.

Estos escolares, 11 hombres y 20 mujeres, fueron estudiados en forma exhaustiva. Se practicó en todos ellos historia clínica minuciosa en cuanto a antecedentes familiares y personales; examen físico general y neurológico, exámenes oftalmológico y otorrinolaringológico; electroencefalogramas. Radiografía de cráneo se tomó sólo en quienes se estimó necesario.

RESUltados. De este estudio, 19 niños ( 9 hombres y 10 mujeres) fueron diagnosticados como jaqueca, y 12 como equivalente convulsivo ( 2 hombres y 10 mujeres).

Los exámenes físico general y neurológico estuvieron dentro de los límites normales. Los exámenes oftalmológico y otorrino presentaron en pocos casos alteraciones que no tenían relación directa con la cefalea motivo de la consulta. En los pacientes en que se solicitó radiografía de cráneo, ésta fue normal.

- De los 19 escolares jaquecosos (3 presentaban jaqueca típica y 16 atípica), 11 acusaban antecedentes de uno o más familiares con jaqueca. El cuadro se inició entre los 4 y 6 años de edad en el 55\% de los casos. Las crisis de dolor se presentaron en la mayoría de ellos con un período intercrítico inferior a 15 días. El tiempo de duración del dolor fue de 6 horas en la mayoría de ellos. Las crisis tenían carácter invalidante en 14 de estos niños, debiendo permanecer en cama.

En 10 pacientes el dolor tenía una localización de tipo hemicránea; en 8 de ellos la cefalea era frontal y en 1, difusa.

En 12 de estos 19 niños el cuadro se acompañaba de náuseas $\mathrm{y} / \mathrm{o}$ vómitos; en uno había anorexia; en los 6 restantes estuvo ausente el sindrome gastro-intestinal.

Al investigar los factores antecedentes a las crisis, predominaron los de carácter psíquico (stress, conflictos de la socialización, etc.) : 11 casos.

El dolor durante las crisis fue descrito como púlsatil por 11 niños; opresivo por 3 y punzante por 1 niño. No fue bien precisado por 5 .

El electroencefalograma se informó normal en 14 de ellos y en 5 se encontraron alteraciones inespecíficas.

- Entre los 12 niños diagnosticados como equivalente convulsivo se registraron antecedentes familiares de epilepsia en 3 casos. No se pesquisaron antecedentes mórbidos familiares en 5 niños.

Las crisis dolorosas habían comenzado antes de los 6 años en 4 de los pacientes; en el resto, 8 casos, después de esa edad. Las crisis de dolor se presentaron en casi todos ellos con períodos intercríticos inferiores a 15 dias. La duración del dolor fluctuaba entre 2 y 6 horas en 11 pacientes, excepto en uno que excedía ese tiempo.

E] dolor tenía una localización de tipo frontal en 5 niños. En otros 2 era holocránea $y$, en el resto, difusa.

El cuadro se acompañaba de náuseas y/o vómitos en 4 pacientes, 2 manifestaban anorexia, uno cólicos abdominales y uno mareo.

Antecedentes de convulsiones tenían 4 niños, en uno de los cuaies se manifestó una crisis de Gran Mal durante el estudio.

El dolor fue descrito como pulsátil en 7 de estos pacientes, opresivo en 2 y punzante en 3. Para 7 de los niños el dolor era un factor invalidante que les impedía incluso asistir a clases durante las crisis.

El electroencefalograma informó disrritmia comicial en 11 casos, un caso fue informado como normal.

Comentario. En nuestra casuística de niños con jaqueca, 3 de ellos presentaban jaqueca típica que se caracteriza por: cefalea recurrente, antecedentes familiares jaquecosos, hemicránea y sindrome gastrointestinal (7 - 10).

Jaqueca atípica o común se diagnosticó en 16 niños. En este cuadro, la cefalea periódica no necesariamente va acompañada por una localización hemicránea del dolor, por sindrome gastrointestinal, y/o antecedentes de jaqueca en la familia ( 7 - 14).

Todos nuestros pacientes catalogados como equivalente convulsivo presentaban también cefalea periódica de diversa localización, asociada o no a síndrome gastrointestinal (3-5-13). (Tablas No 1 y 2 ).

El factor antecedente de las crisis dolorosas, tanto en la jaqueca como en el equivalente convulsivo, fue mayoritariamente el stress emocional. El carácter invalidante del dolor también se dio en proporciones similares para ambos cuadros.

Como se aprecia, la sintomatología entre jaqueca y equivalente convulsivo es similar, especialmente cuando la jaqueca es atípica (3-7).

Será entonces de gran importancia realizar el diagnóstico diferencial.

Si corisideramos que el Equivalente Convulsivo es una epilepsia, su diagnóstico de- 
T A B L A No 1

LOCALIZACION DEL DOLOR EN 31 ESCOLARES CON CEFALEA, SEGUN DIAGNOSTICO. SANTIAGO, 1975.

\begin{tabular}{lcc}
\hline Localización dolor & Jaqueca & Equiv. Conv. \\
\hline Hemicránea & 10 & - \\
Frontal & 8 & 5 \\
Holocránea & - & 2 \\
Otros & 1 & 5 \\
\hline T o t a l & 19 & 12 \\
\hline
\end{tabular}

T A B L A N 2

SINTOMAS ACOMPAÑANTES DE LAS CRISIS DOLOROSAS, EN 31 ESCOLARES SEGUN DIAGNOSTICO. SANTIAGO, 1975

\begin{tabular}{lcrcr}
\hline Sint. acompañantes & Jaqueca & $\%$ & Eq. conv. & $\%$ \\
\hline Náuseas y/o vómitos & 12 & $\mathbf{6 3 , 2}$ & 4 & 33,3 \\
Anorexia & 1 & 5,3 & 2 & 16,7 \\
Dolores abdominales & - & - & $\mathbf{1}$ & 8,3 \\
Mareos & - & - & 1 & 8,3 \\
No presentan & 6 & 31,5 & 4 & 33,3 \\
\hline T o t a 1 & $\mathbf{1 9}$ & $\mathbf{1 0 0 , 0}$ & 12 & 99,9 \\
\hline
\end{tabular}

T A B L A No 3

RESULTADOS ELECTROENCEFALOGRAMA SEGUN DIAGNOSTICO. SANTIAGO, 1975

\begin{tabular}{lcc}
\hline E. E. G. & Jaqueca & Eq. conv. \\
\hline Normal & 15 & 1 \\
Disrritmia comicial & - & 11 \\
Alteraciones inespecíficas & 4 & - \\
\hline T o t a 1 & 19 & 12 \\
\hline
\end{tabular}

berá hacerse sólo cuando exista absoluta certeza (3-9-13). De sobra conocemos las implicancias que este diagnóstico conlleva para el tratamiento del cuadro, que deberá ser de larga duración.

Por lo tanto, cuando un niño ha consultado por cefalea periódica, para diagnosticar un equivalente convulsivo creemos debe exigirse un electroencefalograma anormal con signos claros de actividad comicial intercrítica, con o sin antecedentes de crisis convulsivas ( 11 de nuestros pacientes); o un elec- troencefalograma normal asociado a antecedentes de crisis de Gran Mal ( 1 de nuestros casos). Sólo como elementos de apoyo, es de importancia considerar: antecedentes familiares epilépticos; una localización del dolor no hemicránea y/o ausencia de antecedentes jaquecosos en los familiares.

Edad, sexo, frecuencia de las crisis, característica del dolor, el carácter invalidante del mismo, sindrome gastrointestinal y examen neurológico normal, no tendrían importancia para el diagnóstico diferencial entre jaqueca y equivalente convulsivo, ya que se dieron en relación estadística no significativa para ambos cuadros. Un electroencefalograma con alteraciones inespecíficas tampoco es suficiente para catalogar una cefalea recurrente como de origen epiléptico.

Las alteraciones inespecíficas -que podrían deberse a una inmadurez bioeléctrica del cerebro infantil, que se normaliza con el tiempo- también han sido explicadas para la jaqueca $(10-11)$.

La literatura menciona la reoencefalografía como un elemento útil para el diagnóstico diferencial, aplicada durante las crisis. Este método detectaría vasoconstricción y/o vasodilatación durante las crisis jaquecosas; en cambio estas alteraciones vasculares estarían ausentes en las crisis de equivalente convulsivo. (13).

En la jaqueca, el sustrato anatómico y fisiopatológico del dolor de cabeza estaría explicado por la teoría vascular-humoral (26-15). No sucede lo mismo ccn el equivalente convulsivo, considerado como epilepsia: "fenómenos clínicos que resultan de una descarga neuronal hipersincrónica autosostenida" (9). Esta descarga neuronal excesiva no sería capaz de explicar por sí misma la cefalea, ya que desde Wolf se acepta que el tejido cerebral es indoloro, aún estimulándolo (6-16).

Para explicar la patogenia de la cefalea en el Equivalente Convulsivo, y ésto sólo en el plano de la teoría, nos parece que la descarga neuronal actuaría como gatillo en la liberación de algún tipo de "sustancias productoras de dolor". Estas ejercerían su efecto a nivel de las terminaciones nerviosas presentes en las capas adventicias de los vasos cerebrales.

Finalmente debemos agregar que cuadros clínicamente tan semejantes como jaqueca y equivalente convulsivo, deben diagnosticarse en forma precisa. Como entidades nosológicas distintas, su evolución, posibilidades de tratamiento y pronóstico, son también distintos. 
Se realiza un estudio comparativo entre 19 niños diagnosticados como jaqueca y 12 diagnosticados como Equivalente Convulsivo, seleccionados de un estudio prospectivo de 45 escolares que consultaron por cefalea tenaz y recurrente.

Se encuentra una sintomatología básica similar para ambos cuadros en lo referente a sexo, sindrome gastrointestinal, edad de inicio, frecuencia y caracteristica de la crisis dolorosas.

Electroencefalograma alterado (actividad comicial intercritica), o E. E. G. normal con antecedentes de crisis convulsivas serian elementos esenciales para diagnosticar una cefalea periódica como Equivalente Convulsivo.

Antecedentes familiares de epilcpsia o jaqueca, servirán como apoyo al diagnóstico diferencial.

Se hace un intento para explicar la patogenia de la cefalea en el Equivalente Convulsivo.

Dado que jaqueca y equivalente convulsivo aparecen clinicamente como tan semejantes - siendo entidades nosológicas distintas- se insiste en la necesidad de realizar un correcto diagnóstico diferencial. En ambos cuadros la evolución, tratamiento y pronóstico son diferentes.

\section{SUMMARY}

A comparative study was performed betwcen 19 children diagnosed as having Migraine and 12 patients diagnosed as Convulsive Equivalent, These patients were selected among 45 school age children that were controlled for chronic recurrent cephalaigia.

The basic symptoms are found to be similar regarding sex, gastrointestinal syndrome, age of ouset, frequency and charasteristics of the crisis of pain.

Basic requirements to diagnose a periodic cephalalgia as Convulsive Equivalent are an altered E. E. G. (intercritic comicial activity) or a normal E. E. G. plus a story of convulsive siezures.

A family history of epilepsy or migraine are helpfut in the differential diagnosis.

An attempt is made to explain the pathogenesis of cephalalgia in Convulsive Equivalent.

Considering that Migraine and Convulsive Equivalent appear clinically similar being different diseases, emphasis is made on the correct differential diagnosis. In both diseases the evolution, treatment and prognosis sis are different.
1.--Ajuriaguerra, J.: Manual de Psychiatrie de l'enfant. Masson y Cie. Paris, 1970.

2.-Antony, M.; Hinterberger, H.; Lance J.: Plasma Serotonin in Migraine and Stress. Arch. Neurol., 16: 544, 1967.

3.-Chao, D.; Sexton, J. A; Davis, S. D.: Convulsive Equivalent Syndrome of Childhood. The Journal of Pediatrics 64: 499, 1964.

4.-Devilat, M.; Napolitano, E.; Cox, J.: Equivalentes Convulsizos. ¿Una forma de Epilepsia? Rev. Chilena de Peciatría. 95: 52, 1974.

5.-- Espadaler Medina, J. M.: Cephalées Epiléptiques. World Nelirology, 2: 309, 1961.

6.- Fisher, C. M.: Headache in Cerebrovascular Disease. Handbook of Clinical Neurology, Vol. 5, 1968.

7.-- Friedman, A. P.: The Infinite Variety of Migraine. In Background to Migraine. W. Heiremann, Medical Books Ltd., Londres, 1970.

8.--Friedman, A. P.; Vincken, P.; Bruyan, G. W.: Drug Treatment of Migraine Handbook Clinical Neurology 5: 96, 1968.

9.-Gastaut, H. y col.: Diccionario de Epilepsia Parte 1 Definicionss. O. M. S., Ginebra, 1973.

10.-Heyck, H. Dolor de cabeza. Ed. Marín y Cía,, Barcelona, 1959.

11.-Koch, Ch.: Melchior, J.: Headache in Chilhood. Danish Medical Bulietin, 16: 109, 1969.

12.-Lennox, W. G.: Epilepsy and related disorders. Little Bronwn Co., Boston, 1960.

13.-Poch, G.; Martin, A.; Zavala, H. Ce aíeas epilépticas puras. Cefaleas y Jaquecas. Eudeba, Bs. As., 1973.

14.-Serani, H.; Guzmán, R. Jaquecas atípicas. Rev. Méd. de Chile, 77: 634, 1949.

15.- Sicuteri, Federico: Interpretación "Seca y Húmeda" de las cefoieas esenciales. Cefaleas y Jaquecas. Eudeba. Bs. As., 1973.

16.-Wolf, H. G.: Headache and other Head Pain. Oxford Univ. Press, 1963.

17.-Zanjanian, M. H.: A. Review of Headaches in Children. The Journal of the Medical Society of New Jerscy. 71: 755, 1974. 